\section{THU0598 CAN YOU HEAR THE JOINTS CRYING? MUSCULOSKELETAL EXAMINATION IN JUNIOR DOCTORS' MEDICAL ADMISSION CLERKINGS}

F.Y.H. Kwok, L.H. Lee, A. Gupta. Nottingham University Hospitals NHS Trust, Nottingham, United Kingdom

Background: Physical examination is an indispensable skill in the art of medicine which, together with history taking, enables physicians to achieve a clinical diagnosis. Previous studies have consistently shown wide variations in the documentation of physical examination findings on systemic review $(1,2)$. In particular, musculoskeletal examination was found to be frequently omitted. Objectives: The aim of this study was to evaluate the quality of junior doctors' medical admission clerking, with a focus on clinical examination.

Methods: Fifty acute medical admission clerking entries by junior doctors in a university hospital were reviewed. Case notes were assessed for clinical details and physical examination performed.

Results: Mean age was 69. The commonest presenting complaint was fall or collapse. Musculoskeletal examination was only documented in $24 \%$ of admitted patients. There was disparity in the quality of documentation, ranging from complete omission to comments such as "valgus deformity", "erythema", "limited abduction" and "unable to SLR". Overall, the documentation of cranial nerves examination, limb neurological examination, abbreviated mental test and Glasgow coma scale was less robust (40\%, 52\%, $48 \%$ and $66 \%$ respectively). The documentation of musculoskeletal and neurological examination contrasts markedly with other systems: respiratory system $100 \%$, cardiovascular system $98 \%$ and abdominal system $98 \%$.

Conclusions: The omission of musculoskeletal examination may reflect a general apathy towards musculoskeletal health. Despite fall or collapse being the most common reason for medical admission in this study, musculoskeletal examination was only documented in a minority of patients. With an aging patient population, it is vital for physicians to address musculoskeletal disorders which are likely to be more prevalent on the acute medical take. Junior doctors' lack of confidence in performing musculoskeletal examination may also be another contributing factor. It is important that junior doctors are provided feedback on their clinical assessment by senior doctors when essential components of examination are omitted. National training programmes should consider including these areas in postgraduate curriculum to ensure competency is achieved.

\section{References:}

[1] ) Doherty M, Abawi J, Pattrick M. Audit of medical inpatient examination: a cry from the joint. J R Coll Physicians Lond 1990;24(2):115-8.

[2] ) Myers A, McDonagh JE, Gupta K, et al. More "cries from the joints": assessment of the musculoskeletal system is poorly documented in routine paediatric clerking. Rheumatology (Oxford) 2004;43(8):1045-9.

Disclosure of Interest: None declared

DOI: 10.1136/annrheumdis-2017-eular.2602

\section{THURSDAY, 15 JUNE 2017 \\ Public health, health services research and health economics}

\section{THU0599 BENEFIT RISK RATIO FOR BIOLOGICAL AGENTS IN JUVENILE IDIOPATHIC ARTHRITIS, A META-ANALYSIS OF RANDOMIZED CLINICAL TRIALS}

N. Cabrera ${ }^{1}$, P. Janiaud ${ }^{1}$, A. Belot ${ }^{2}$, B. Kassai ${ }^{1,3}$, J.-C. Lega ${ }^{1} .{ }^{1}$ UMR 5558 CRNS Lyon; ${ }^{2}$ University of Lyon $1 ;{ }^{3}$ Clinical Investigation Center/CIC 1407 , INSERM, Lyon, France

Background: The biological agents (BAs) have revolutionized the care and have improved the prognosis of juvenile idiopathic arthritis (JIA). Effectiveness and short-term tolerance was well established in randomized clinical trials (RCTs), initially in JIA. However, there is an insufficient evidence for the long-term tolerance ${ }^{1}$. The serious adverse events (SAEs) include infections, malignancies and drug induced auto-immune diseases (e.g. uveitis)

Objectives: To assess the benefit/risk balance of BAs assessed by RCTs vs placebo or vs standard treatment in JIA, using meta-analysis (MA) technique. Methods: All RCTs in JIA comparing BAs to placebo or standard treatment (e.g. methotrexate) published between 1950 and February 2016 were eligible. Data source: Cochrane, Medline, ClinicalTrial.gov register. The ILAR classification for $\mathrm{JIA}^{2}$ was used and the clinical efficacy of treatment was measured by the ACR pediatric score ${ }^{3}$. Efficacy was analyzed considering the design of study. Effectiveness (ACRpedi30) was estimated as the measure of the benefit of the BAs and SAEs as a measure of risk by random effect models. The benefit/risk balance was analyzed using the net efficacy adjusted for risk (NEAR) ${ }^{4}$. An OR $>1$ indicates that the treatment has a beneficial effect and $O R<1$ a deleterious effect. Subgroups analyses were made to account the heterogeneity of JIA. We explored potential heterogeneity by subgroups analysis according with BAs and JIA subtypes. Results: We included 20 RCTs conducted in JIA encompassing 1533 children. The disease duration, at the inclusion of RCTs, varies between 2 and 6 years for most studies. The maintenance of the therapeutic effect was estimated in the studies using withdrawal design in 6 studies. The maintenance of clinical response showed a large heterogeneity. Sub-groups analyses showed that the heterogeneity is marked in the systemic $\mathrm{JIA}^{5}$. The global NEAR OR was in favor of BAs in parallel (OR 3.83, Cl 1.49-9.82) and withdrawal (OR 2.75, Cl 1.51-5.01) trials. The efficacy of BAs in JIA was superior to the placebo in parallel (OR 5.46, Cl 2.26-13.21) and withdrawal (OR 3.52, Cl 2.15-5.77) trials. In RCTs (parallel and withdrawal design), SAEs did not differ between BAs and control (OR 1.18, $\mathrm{Cl}$ 0.73-1.9). No death occurred at follow-up.

Conclusions: This is the first MA assessing all BAs used in all JIA categories combined. This MA in patients with JIA shows that the benefit/risk ratio of BAs in JIA is favorable.

\section{References:}

[1] Klotsche, J. et al. Long-term safety of etanercept and adalimumab compared to methotrexate in patients with juvenile idiopathic arthritis (JIA). Ann. Rheum Dis. 75, 855-861 (2016)

[2] Petty, R. E. et al. International League of Associations for Rheumatology classification of juvenile idiopathic arthritis: second revision, Edmonton, 2001. J. Rheumatol. 31, 390-392 (2004)

[3] Consolaro, A. et al. Optimisation of disease assessments in juvenile idiopathic arthritis. Clin. Exp. Rheumatol. 32, S-126-130 (2014).

[4] Boada, J. N. et al. Net efficacy adjusted for risk (NEAR): a simple procedure for measuring risk:benefit balance. PloS One 3, e3580 (2008).

[5] Tarp, S. et al. Efficacy and safety of biological agents for systemic juvenile idiopathic arthritis: a systematic review and meta-analysis of randomized trials. Rheumatol. Oxf. Engl. 55, 669-679 (2016).

Disclosure of Interest: None declared

DOI: 10.1136/annrheumdis-2017-eular.4568

\section{THU0600 CUMULATIVE ADVERSE CHILDHOOD EXPERIENCES ARE ASSOCIATED WITH POOR OUTCOMES IN ADULTS WITH SYSTEMIC LUPUS ERYTHEMATOSUS}

K. DeQuattro $^{1}{ }^{\text {, L. Trupin }}{ }^{1}$, P.P. Katz ${ }^{1}$, C. Lanata ${ }^{1}$, E.H. Yelin ${ }^{1}$, L.A. Criswell ${ }^{1}$, C.G. Helmick ${ }^{2}$, M. Dall'Era ${ }^{1}$, J. Yazdany ${ }^{1} .{ }^{1}$ Division of Rheumatology, University of California San Francisco, San Francisco; ${ }^{2}$ Division of Rheumatology, Centers for Disease Control and Prevention, Atlanta, United States

Background: Adverse childhood experiences (ACE) such as abuse, neglect and household challenges are associated with poorer adult health status and onset of rheumatic diseases. There has been no research associating ACE with outcomes among adults with systemic lupus erythematosus (SLE).

Objectives: To characterize relationships of ACE and health outcomes of disease activity, damage, quality of life and depression in SLE patients.

Methods: Data were derived from the California Lupus Epidemiology Study (CLUES), a population based, multi-ethnic cohort of patients with SLE. Participants completed self-report measures of SLE activity (Systemic Lupus Activity Questionnaire; SLAQ), damage (Brief Index of Lupus Damage; BILD), quality of life (SF-36), depression (Patient Health Questionnaire; PHQ8) and sociodemographics. They completed the Adverse Childhood Experiences (ACE) survey, a validated 10 -item scale covering 3 domains (abuse, neglect and household challenges prior to age 18). We compared demographics and SLE outcomes by ACE score and domains using ANOVA.

Results: The 166 CLUES participants were mostly women (89\%) and were racially/ethnically diverse (31\% non-Hispanic White, 22\% Hispanic, 15\% African American, $31 \%$ Asian American). Mean age was $44 \pm 14$; mean age at diagnosis $28 \pm 12$. The median ACE score was 1; $30(18 \%)$ had a score of 4 or higher. ACE scores $\geq 4$ were more common in Hispanic (27\%) and African American (32\%) participants $(p=0.01)$ compared to other races/ethnic groups, and in participants with poverty level incomes ( $61 \%$ vs $13 \%, p<0.001)$; but did not differ by education or age at study entry or diagnosis. Higher overall ACE scores were associated with greater SLE activity and damage, poorer quality of life, and higher levels of depressive symptoms. For each ACE domain, increasing scores were generally associated with worse outcomes, but did not always reach statistical significance (Table).

Table 1. SLE Outcomes by Adverse Childhood Event (ACE) Scores and Domains

\begin{tabular}{lcccccc}
\hline & Score & $\mathrm{n}$ & \multicolumn{4}{c}{ Outcomes [mean (sd)] } \\
\cline { 4 - 7 } & & & SLAQ & BILD & SF36PCS & PHQ8 \\
\hline Total ACE score & 0 & 66 & $6.0(6.2)$ & $1.7(2.0)$ & $46.0(10.5)$ & $4.3(4.1)$ \\
& 1 & 31 & $6.9(5.4)$ & $1.4(1.5)$ & $44.3(8.5)$ & $4.8(4.0)$ \\
& $2-3$ & 39 & $11.2(7.6)$ & $1.7(2.1)$ & $41.0(10.5)$ & $7.8(5.6)$ \\
& $4+$ & 30 & $11.8(8.0)$ & $3.0(2.9)$ & $38.1(11.1)$ & $7.3(4.6)$ \\
Household Challenges & & p-value & $<0.001$ & 0.02 & $<0.001$ & 0.003 \\
& $0^{*}$ & 66 & $6.0(6.2)$ & $1.7(2.0)$ & $46.0(10.5)$ & $4.3(4.1)$ \\
& 1 & 33 & $9.1(6.6)$ & $1.8(2.2)$ & $42.3(8.8)$ & $6.3(5.7)$ \\
Neglect & $2+$ & 24 & $11.5(8.0)$ & $2.4(2.2)$ & $40.3(10.6)$ & $7.6(5.1)$ \\
& & $p-v a l u e$ & 0.002 & 0.35 & 0.04 & 0.01 \\
& $0^{*}$ & 66 & $6.0(6.2)$ & $1.7(2.0)$ & $46.0(10.5)$ & $4.3(4.1)$ \\
Abuse & 1 & 33 & $11.6(7.0)$ & $2.4(2.7)$ & $37.7(9.9)$ & $8.1(4.8)$ \\
& $2+$ & 24 & $9.8(7.4)$ & $1.6(0.9)$ & $42.2(5.2)$ & $6.4(2.6)$ \\
& & $p-v a l u e$ & $<0.001$ & 0.33 & 0.001 & $<0.001$ \\
& $0^{*}$ & 66 & $6.0(6.2)$ & $1.7(2.0)$ & $46.0(10.5)$ & $4.3(4.1)$ \\
& 1 & 26 & $10.1(6.3)$ & $1.7(1.9)$ & $38.8(10.4)$ & $8.1(4.5)$ \\
& $2+$ & 26 & $13.9(7.6)$ & $3.0(3.2)$ & $37.5(10.6)$ & $6.9(4.5)$ \\
& & $p-v a l u e$ & $<0.001$ & 0.04 & $<0.001$ & $<0.001$ \\
\hline
\end{tabular}

*Zero-level excludes respondents with scores in other ACE domains. 